\title{
Когерентность конденсата поляритонов в планарных микрорезонаторах в магнитном поле
}

\author{
(C) А.В. Черненко ${ }^{+}$, А. Рахими-Иман*凶, Ю. Фишер*, М. Амтор*, К. Шнайдер*, \\ С. Райзенштайн ${ }^{* \nabla}$, А. Форхель*, С. Хёфолинг* \\ + Институт физики твердого тела Российской академии наук, \\ 142432 Черноголовка, Россия \\ * Technische Physik, Physikalisches Institut and Wilhelm Conrad Röntgen Research Center \\ for Complex Material Systems, Universitat Würzburg, \\ D-97074 Würzburg, Germany \\ $\Delta$ Department of Physics and Materials Sciences Center, Philipps-Universität Marburg, \\ D-35032 Marburg, Germany \\ $\checkmark$ Institut fur Festkörperphysik, Technische Universität Berlin, \\ D-10623 Berlin, Germany \\ E-mail: chernen@yandex.ru
}

(Получена 27 апреля 2016 г. Принята к печати 10 мая 2016 г.)

B планарных GaAs-микрорезонаторах в магнитном поле до 5 Тл, перпендикулярном плоскости роста структур, в условиях резонансной импульсной накачки в точку, близкую к точке перегиба нижней дисперсионной кривой, наблюдается зеемановское расщепление спиновых подуровней конденсата поляритонов. Оно сопровождается значительным изменением степени циркулярной поляризации и коррелятора 2-го порядка $g^{2}(0)$. Оказалось, что коррелятор отличается для расщепленных в магнитном поле спиновых подуровней поляритонного конденсата. В частности, измерения коррелятора свидетельствуют о различии в порогах конденсации для спиновых подуровней. Изначально отличающиеся в отсутствие поля значения коррелятора растут, достигая максимального значения, а затем уменьшаются и сравниваются между собой для разных поляризаций в поле 5 Тл.

\section{1. Введение}

Наличие у поляритонов спина приводит к тому, что свойства поляритонного конденсата существенно отличаются от свойств конденсата безспиновых частиц, и ответственно за ряд новых эффектов. В первую очередь это связано с поляритон-поляритонным взаимодействием, которое зависит от взаимной ориентации спинов поляритонов таким образом, что частицы с сонаправленными спинами отталкиваются, тогда как между частицами с противоположно направленными спинами существует слабое притяжение [1].

Поляритон-поляритонное взаимодействие приводит к интересному эффекту, предсказанному в [2], - подавлению зеемановского расщепления поляритонных уровней в слабых магнитных полях в условиях термодинамического равновесия. Это предсказание породило значительный интерес к магнитооптическим исследованиям конденсата поляритонов. В работе [3] докладывалось об экспериментальном наблюдении подавления зеемановского расщепления, однако при этом поведение степени циркулярной поляризации фотолюминесценции (ФЛ) не соответствовало теории. Дальнейшие исследования показали, что еще одним существенным фактором, влияющим на поведение поляритонной системы в магнитном поле, является скорость спиновой релаксации. Например, отсутствие спинового равновесия между расщепленными магнитным полем спиновыми подуровнями системы поляритонов может привести к изменению знака зеемановского расщепления, что наблюдалось в работе [4]. Этот эффект оказалось возможным объяснить, предположив отсутствие равновесия между спиновыми подуровнями конденсата. Однако предложенная в [4] модель позволила получить лишь качественное согласие между экспериментом и теорией, что указывает на то, что исследования свойств неравновесного конденсата в магнитном поле сохраняют свою актуальность. В этой связи стоит упомянуть работу [5], где предлагается интерферометр для детектирования фазы Берри, а также работу [6], в которой предсказывается зависимость свойств полувихрей в поляритонном конденсате от магнитного поля. Эти предсказания пока не нашли экспериментальной проверки.

В данной работе, в дополнение к измерениям ФЛ, представлены результаты исследований когерентных свойств конденсата поляритонов и анализ зависимости коррелятора 2-го порядка $g^{(2)}(0)$ от магнитного поля. Измерения коррелятора представляют интерес, поскольку являются независимым от ФЛ источником информации о свойствах конденсата поляритонов.

\section{2. Эксперимент}

Для изучения поведения конденсата поляритонов в магнитном поле использовались высокодобротные микрорезонаторы $(Q>10000)$, содержащие 4 GaAs-квантовые ямы толщиной 13 нм, окруженные AlAs-барьерами по 4 нм каждый, которые были помещены в пучность $\lambda / 2$ AlAs-микрорезонатора, содержащего 23/27 пар брэгговских зеркал $\mathrm{AlAs} / \mathrm{Al}_{0.2} \mathrm{Ga}_{0.8} \mathrm{As}$. Расщепление Раби для этой структуры составляет $\Omega_{0}=10.5$ мэВ. 
Для резонансного возбуждения образца в точку вблизи точки перегиба нижней поляритонной (НП) ветви использовался титан-сапфировый лазер с длительностью импульсов 5 пс и частотой повторений 82 МГц. Луч лазера фокусировался на образец под углом $\sim 15^{\circ}$, близким к „магическому“, что соответствует продольному квазиимпульсу $k_{\|} \approx 1.8$ мкм $^{-1}$. Режима конденсации удалось добиться при малых положительных расстройках резонатора $\delta=E_{x}-E_{c} \approx 1-2$ мэВ между уровнем экситона в ямах $E_{x}$ и энергией оптической моды резонатора $E_{c}$. Излучение лазера фокусировалось в пятно на поверхности образца диаметром $\sim 5$ мкм.

Измерения микрофотолюминесценции проводились в криостате, оснащенном сверхпроводящим магнитном, в магнитном поле до 5 Тл при температуре $5 \mathrm{~K}$ как в реальном, так и в фазовом пространствах. Пятно возбуждения газа поляритонов, наблюдаемое в реальном пространстве, было высокой степени однородности, а для конденсата оно не обладало заметной асимметрией.

\section{3. Зеемановское расщепление, поляризация и диамагнитный сдвиг}

Образование конденсата поляритонов в микрорезонаторе при усредненной по времени плотности накачки, превышающей критическую $P>P_{\text {th }}=6.54 \mathrm{\kappa BT} / \mathrm{cm}^{2}$, подтверждается (i) существенным сужением распределения поляритонов на НП дисперсионной кривой, (ii) фиолетовым сдвигом $\sim 0.5$ мэВ НП ветви, (iii) появлением интерференционной картины в эксперименте Юнга, подобно описанному в [7], (iv) пороговым возникновением отсчетов лавинных диодов в интерферометре Хэнбери-Брауна-Твисса (ХБТ). Известно, что, в силу нелинейного роста интенсивности при превышении порога конденсации, отсчеты диодов ниже порога конденсации не превышают уровень шума [7].

Характерные спектры ФЛ газа поляритонов при накачках как выше, так и ниже пороговой в нашем случае аналогичны приведенным во многих работах, например в [4]. Поскольку свойства газа поляритонов НП ветви в магнитном поле исследовались во многих работах, в частности в работе [4], мы не будем останавливаться на них подробно. Характерные спектры ФЛ образца в $\sigma^{ \pm}$-поляризациях при накачках выше пороговой в поле $B=5$ Тл приведены на рис. 1. На них видно, что образование конденсата поляритонов сопровождается скачкообразным изменением степени циркулярной поляризации, $\rho_{c}$, от 0 до $80 \%$ в отсутствие поля (см. рис. 2). С ростом поля циркулярная поляризация падает и достигает $\rho_{c} \approx 0 \%$ в поле 5 Тл. При плотности возбуждения ниже пороговой степень циркулярной поляризация близка к $0 \%$ в любом магнитном поле.

В плоском микрорезонаторе или микростолбиках цилиндрической формы ФЛ конденсата линейно поляризована, со степенью линейной поляризации, которая может

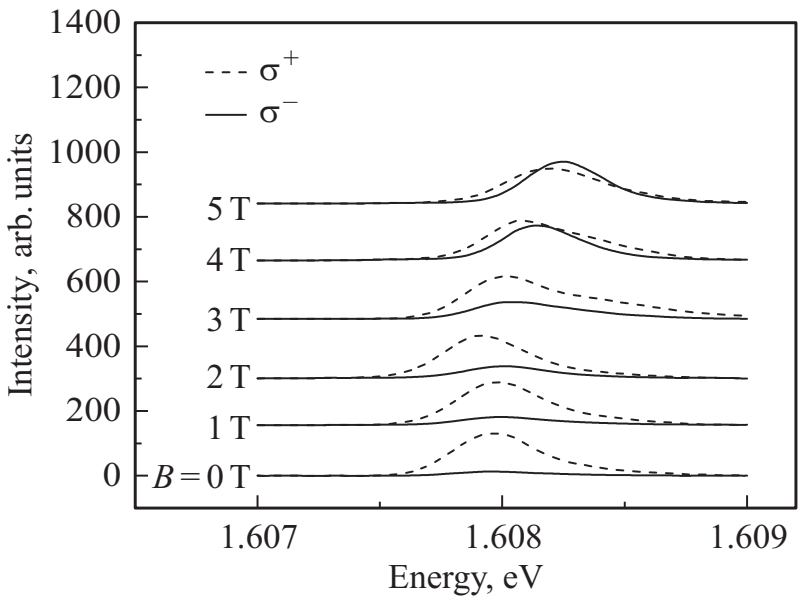

Рис. 1. Спектры фотолюминесценции образца в магнитном поле 0-5 Тл, записанные в обеих циркулярных поляризациях при постоянной плотности накачки $P=1.2 P_{\text {th }}$, где $P_{\text {th }}=6.54 \mathrm{\kappa BT} / \mathrm{cm}^{2}$.

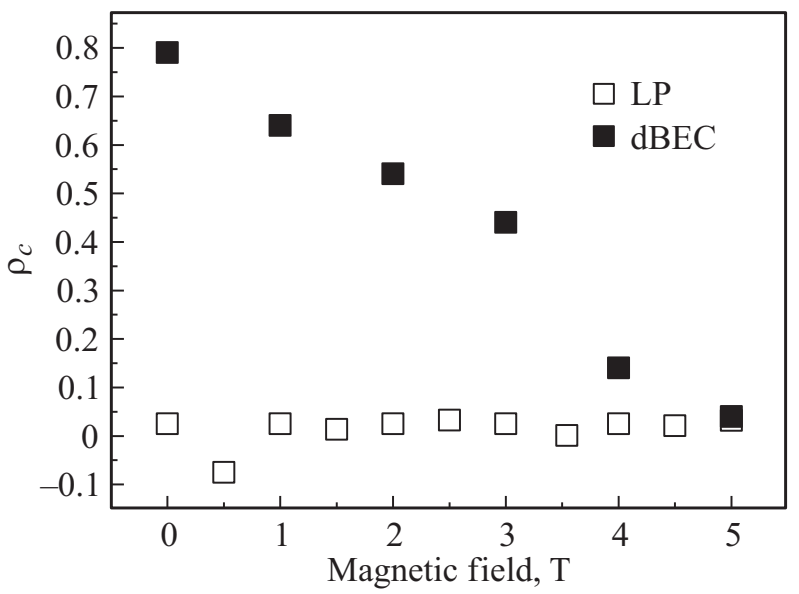

Рис. 2. Зависимость степени циркулярной поляризация ФЛ НП ветви $\rho_{c}$ в магнитном поле для газа поляритонов $(L P)$ и динамического конденсата $(d B E C)$ при постоянной накачке $P=1.2 P_{\text {th }}$

превышать 90\% [8,9]. Это является следствием спинзависимого взаимодействия между поляритонами, так как энергия конденсата минимальна при равном заполнении уровней спин-поляризованных уровней [8]. Вместе с тем ненулевая циркулярная поляризация конденсата поляритонов в случае импульсной накачки наблюдалась ранее в работе [10]. Согласно модели, предложенной в [11], это связано с различной эффективностью рассеяния поляритонов разной поляризации в точку $k=0$ мкм $^{-1}$. Условия возбуждения таковы, что лазерный луч падает на образец под углом, что делает свет, возбуждающий поляритоны в микрорезонаторе, слабо эллиптически поляризованным. Это, в свою очередь, ведет к различию в уровнях заселенности спин-поляризованных компонент конденсата при возбуждениях выше порога параметрического рассеяния и таким образом одна из 
спиновых компонент конденсата оказывается более заселенной.

Расщепление спин-поляризованных уровней в самом общем случае зависит не только от магнитного поля, но и от разницы заселенностей спиновых подуровней изза поляритон-поляритонного взаимодействия. При плотности накачки меньше пороговой $P<P_{\text {th }}$ оно несущественно, поэтому сдвиг спин-поляризованных уровней с точностью до членов порядка $O\left(\delta / \hbar \Omega_{0}\right)$ в поле $B$ описывается выражением $[4,12]$

$$
\begin{aligned}
E_{L P}^{ \pm}(B) & =E_{L P}(0) \pm 0.5 x g_{x} \mu_{\mathrm{B}} B+B x \chi_{x} B^{2} \\
& = \pm 0.5 \mu_{\mathrm{B}} g_{\mathrm{eff}} B+\chi_{\mathrm{eff}} B^{2}
\end{aligned}
$$

где $x=|X(\delta(B))|^{2}$ - экситонная доля поляритонов, а $X(\delta, B)$ - коэффициент Хопфилда, $n^{ \pm}-$плотности поляритонов в точке $k=0$ НП дисперсионной кривой с соответствующими проекциями момента экситона $| \pm 1\rangle$ на ось $0_{z}, E_{L P}(0)$ - энергия поляритона в нулевом поле, $g_{x}-g$ - фактор экситона, $\chi_{x}$ - диамагнитная восприимчивость экситона, $E_{L P}(0)$ - энергия поляритона в нулевом поле, $\mu_{\mathrm{B}}-$ магнетон Бора. Коэффициент $|X(\delta(B))|^{2}$ зависит от магнитного поля из-за диамагнитного сдвига экситонного уровня и может оказаться существенным [4].

Выше порога конденсации $P>P_{\text {th }}$ возрастает роль поляритон-поляритонного взаимодействия, которое зависит от плотности конденсата и в выражении для положения линий появляется дополнительный член:

$$
\begin{aligned}
E_{L P}^{ \pm}(B)= & E_{L P}(0) \pm 0.5 x g_{x} \mu_{\mathrm{B}} B \\
& +\left(\alpha_{1}+\alpha_{2}\right) x^{2} n^{ \pm}+x \chi_{x} B^{2},
\end{aligned}
$$

$n^{+}$- плотности поляритонов в конденсате с соответствующими проекциями момента экситона $| \pm 1\rangle$ на ось $0_{Z}, \mu_{\mathrm{B}}$ - магнетон Бора, $\alpha_{1}, \alpha_{2}-$ константы поляритон-поляритонного взаимодействия [1]. В общем случае $E_{L P}^{+}(B)$ может иметь довольно сложный вид и, кроме поляритон-поляритонного взаимодействия, в конденсате нужно учитывать взаимодействие поляритонов с резервуаром экситонов $[8,13]$. Это взаимодействие может оказаться существенным и привести к новым эффектам, подобным наблюдаемому в работе [13].

В условиях термодинамического равновесия поляритон-поляритонное взаимодействие между спиновыми подуровнями ответственно за подавление зеемановского расщепления [2], при отсутствии равновесия между уровнями оно может приводить к изменению знака зеемановского расщепления [4]. Отсутствие этих эффектов в нашем случае, видимо, связано с интенсивной импульсной накачкой, используемой для возбуждения ФЛ.

Оказалось, что экспериментальные результаты хорошо описываются выражением (2) в пренебрежении поляритон-поляритонным взаимодействием:

$$
E_{L P}^{ \pm}(B)=E_{L P}(0) \pm 0.5 x g_{x} \mu_{\mathrm{B}} B+x \chi_{x} B^{2} .
$$

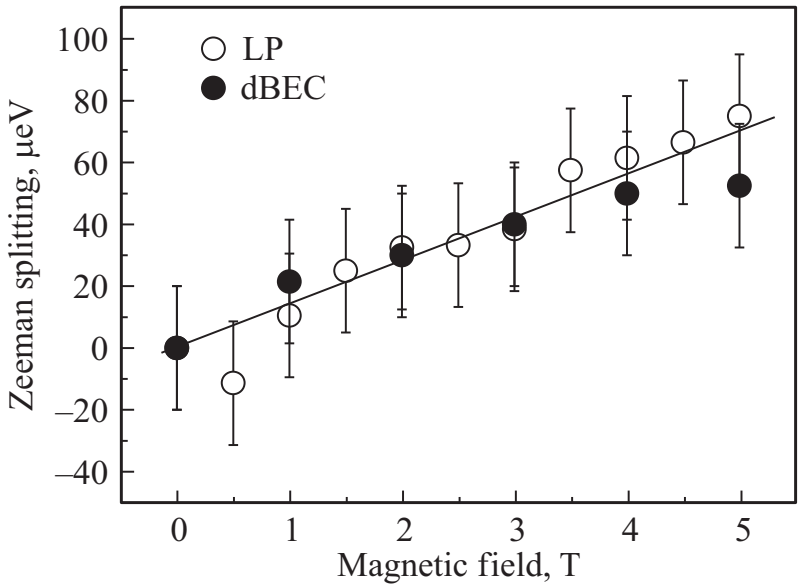

Рис. 3. Зеемановское расщепление линий ФЛ НП при возбуждении ниже $(L P)$ и выше $(d B E C)$ порога конденсации. Подгонка экспериментальных точек с помощью формулы (2) показана прямой линией.

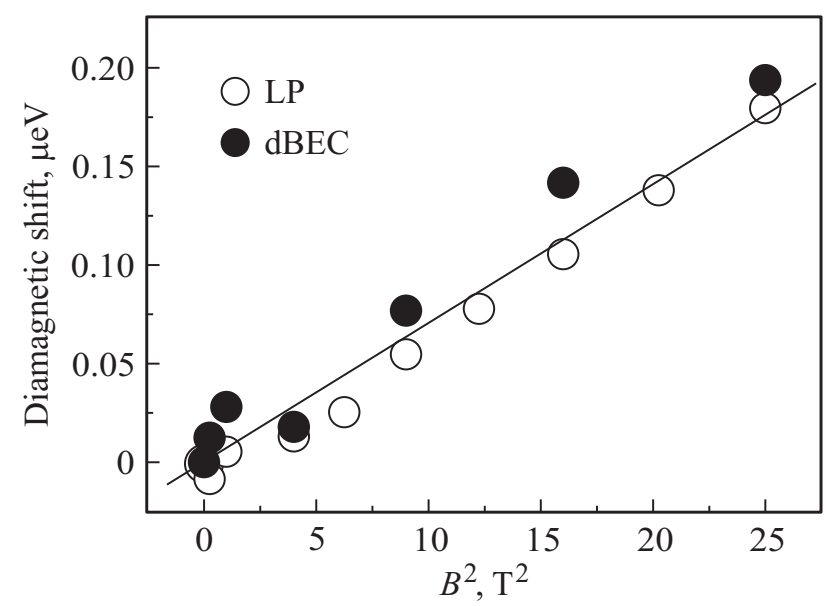

Рис. 4. Величина диамагнитного сдвига НП линии при $k=0$ мкм $^{-1}$, извлеченная из экспериментальных данных при возбуждении ниже $(L P)$ и выше $(d B E C)$ порога конденсации. Результат аппроксимации с помощью формулы (2) представлен прямой линией.

Зависимость зеемановского расщепления уровней конденсата от поля представлена на рис. 3. Величина эффективного $g$-фактора, полученного из экспериментальных данных, представленных на рис. 1, оказалось равной $g_{\text {eff }}=x g_{x}=-1.0 \pm 0.15$. Изменения эффективного $g$-фактора при переходе через порог конденсации, подобные тем, что наблюдались в работах $[3,4]$, не были зафиксированы.

Зависимость диамагнитного сдвига уровней поляритонов от $B^{2}$ представлена на рис. 4. Величина диамагнитной константы, извлеченной из экспериментальных данных, оказалась $\chi_{\text {eff }}=x \chi_{x}=7.0 \pm 0.3$ мкэВ/ТЛ ${ }^{2}$. Экспериментальные результаты не демонстрируют скачка диамагнитного сдвига при переходе через порог конденсации, о котором сообщалось в [4]. 
Кроме поляритон-поляритонного взаимодействия, на зависимость $E_{L P}^{ \pm}(B)$ влияет еще ряд факторов. Так, диамагнитный сдвиг изменяет величину расстройки резонатора $\delta$ и ведет к изменению коэффициентов Хопфилда и эффективной массы, силы осциллятора и дисперсионной кривой. Кроме изменения коэффициента Хопфилда, на силу осциллятора также влияет поджатие волной функции экситона магнитным полем $[14,15]$. Последнее приводит к изменению частоты Раби, поскольку $\Omega_{0} \sim \sqrt{f}$. Однако обнаружение таких тонких эффектов лежит за пределами точности наших измерений.

\section{4. Когерентность второго порядка спин-поляризованного конденсата}

Наряду с измерениями циркулярной поляризации и зеемановского расщепления конденсата поляритонов, были проведены измерения функции когерентности 2-го порядка для обеих циркулярных поляризаций. Для того чтобы измерить коррелятор отдельно для разных поляризаций, сигнал люминесценции пропускался через четвертьволновую пластинку, за которой находились линейный поляризатор и монохроматор. После этого он направлялся в интерферометр Хэнбери-Брауна-Твиса (ХБТ), оснащенный парой быстрых лавинных диодов с характерными временами отклика $\sim 40$ пс. Измерительная система настраивалась на максимум интенсивности спин-поляризованных линий при каждом значении магнитного поля.

Экспериментально измеряемая интерферометром величина $G^{(2)}(0)$ при импульсном возбуждении представляет собой усредненный по времени импульса коррелятор $g^{(2)}(0)[16]$. Результаты измерения $G^{(2)}(0)$ в разных магнитных полях для обеих циркулярных поляризаций представлены на рис. 5. Они получены при плотности мощности возбуждения, соответствующей спектрам на

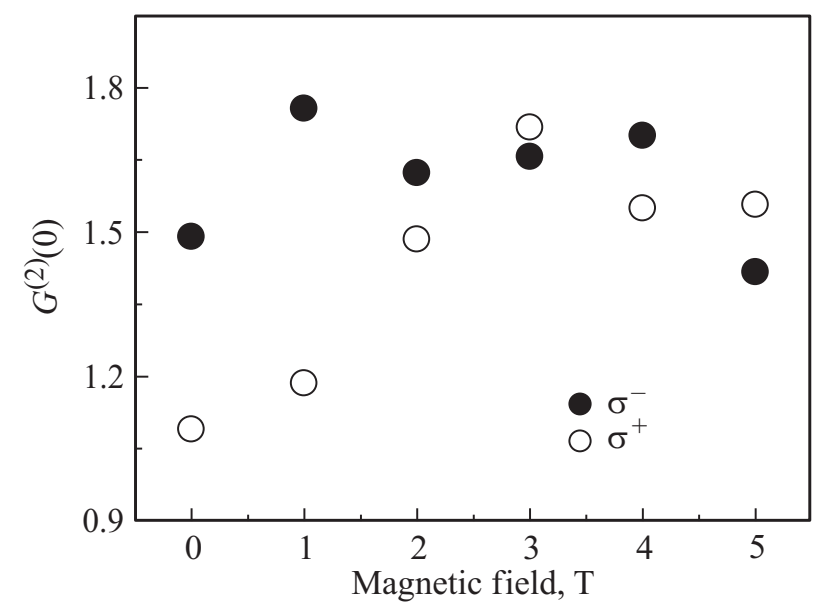

Рис. 5. Зависимость функции когерентности конденсата поляритонов $G^{(2)}(0)$ от магнитного поля при постоянной накачке $P=1.2 P_{\text {th }}$ для обеих циркулярных поляризаций.

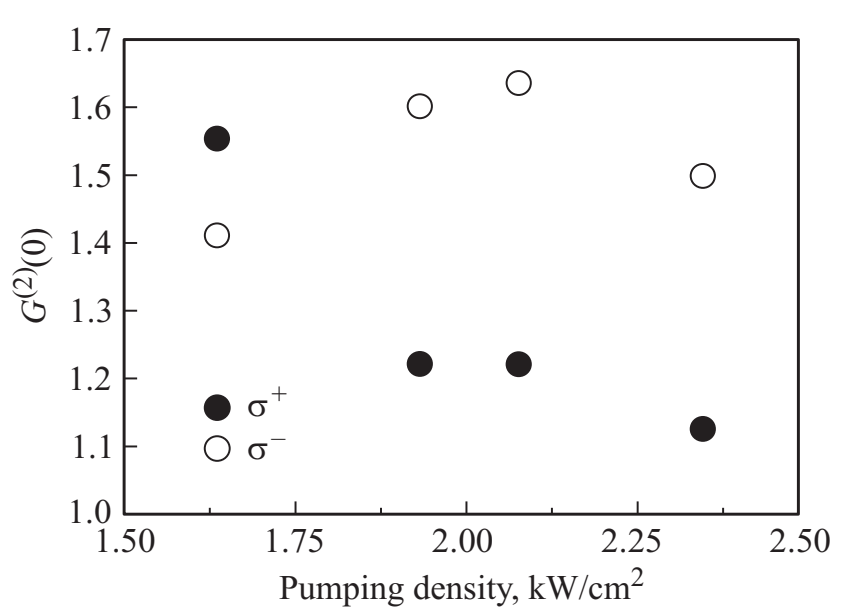

Рис. 6. Зависимость $G^{(2)}(0)$ конденсата от плотности возбуждения в поле $B=5$ Тл.

рис. 1. Линия ФЛ с $\sigma^{+}$-поляризацией и большим числом счетов показывает высокую степень когерентности $G^{(2)}(0) \approx 1.1$, тогда как у $\sigma^{-}$-поляризованной линии $G^{(2)}(0) \approx 1.5$. С ростом магнитного поля $G_{\sigma^{+}}^{(2)}(0)$ растет, в то время как $G_{\sigma^{-}}^{(2)}(0)$ мало изменяется и в поле, большем 3 Тл, так что их значения сравниваются при $B=5$ Тл. Разница в $G^{(2)}(0)$ для разных поляризаций, очевидно, связана с различием в заселенностях, расщепленных полем спиновых подуровней конденсата. Дальнейшее увеличение магнитного поля ведет к уменьшению $G^{(2)}(0)$ для обеих поляризаций,что означает выравнивание населенностей подуровней, параллельно с этим уменьшается степень циркулярной поляризации конденсата, что видно из рис. 2.

Кроме того, величина коррелятора зависит от плотности накачки, как показано на рис. 6 . Величина $G^{(2)}(0)$ уменьшается с ростом плотности оптического возбуждения, что соответствует поведению коррелятора, наблюдаемому в работе [7], и является характерным поведением коррелятора в обычных лазерах. Оно объясняется ростом степени когерентности при росте интенсивности лазерного излучения. Аналогично объясняется тот факт, что более интенсивная линия $\sigma^{+}$демонстрирует более высокую степень когерентности, чем линия $\sigma^{-}$, как это видно на рис. 5. Таким образом, спин-разрешенные измерения $G^{(2)}(0)$ показывают значительную разницу в заселенностях спиновых подуровней конденсата и хорошо коррелируют со степенью циркулярной поляризации.

\section{5. Заключение}

Измерения фотолюминесценции спин-поляризованного конденсата поляритонов в микрорезонаторах в магнитном поле показывают, что выше порога стимулированного поляритон-поляритонного рассеяния, излучение конденсата оказывается циркулярно поляризованным даже при $B=0$ Тл при незначительной степени 
циркулярной поляризации возбуждающего излучения. Измерения коррелятора 2-го порядка предоставляют независимую информацию о свойствах конденсата спинорных поляритонов. Эти измерения показали, что с ростом магнитного поля абсолютная величина степени циркулярной поляризации уменьшается, и вместе с ней исчезает разница в значениях коррелятора 2-го порядка. В постоянном магнитном поле величина коррелятора уменьшается с ростом плотности накачки, что вызвано изменением в заселенностях спин-поляризованных подуровней.

Авторы благодарят В.Д. Кулаковского за обсуждение результатов и ценные замечания.

Работа выполнена при финансовой поддержке РФФИ (грант № 14-02-01233) и правительства земли Бавария.

\section{Список литературы}

[1] A. Kavokin, J. Baumberg, G. Malpuech, F. Laussy. Microcavities (Oxford University Press, 2007).

[2] Y.G. Rubo, A.V. Kavokin, I.A. Shelykh. Phys. Lett. A, 358, 227 (2006).

[3] A.V. Larionov, V.D. Kulakovskii, S. Höefing, C. Schneider, L. Worschech, A. Forchel. Phys. Rev. Lett., 105, 256401 (2010).

[4] J. Fischer, S. Brodbeck, A.V. Chernenko, I. Lederer, A. Rahimi-Iman, M. Amthor, V.D. Kulakovskii, M. Kamp, M. Durnev, A.V. Kavokin, S. Höeffling. Phys. Rev. Lett., 112, 093902 (2014).

[5] A. Shelych, G. Pavlovic, D.D. Solnyshkov, G. Malpuech. Phys. Rev. Lett., 102,046407 (2009).

[6] M.T. Solano, Y. Rubo. Superlat. Microstr., 49, 318 (2011).

[7] A. Rahimi-Iman, A.V. Chernenko, J. Fischer, S. Brodbeck, M. Amthor, C. Schneider, A. Forchel, S. Höefling, S. Reitzenstein, M. Kamp. Phys. Rev. B, 86,155 308 (2012).

[8] V.D. Kulakovskii, A.S. Brichkin, S.V. Novikov, C. Schneider, S. Hoeing, M. Kamp, A. Forchel, N.A. Gippius. Phys. Rev. B, 85, 155322 (2012).

[9] J. Kasprzak, M. Richard, S. Kundermann, A. Baas, P. Jeambrun, J.M.J. Keeling, F.M. Marchetti, M.H. Szymanska, R. Andze, J.L. Stachli, V. Savona, P.B. Littlewood, B. Deveaud, L.S. Dang. Nature, 443, 409 (2006).

[10] H. Deng, G. Weihs, C. Santori, J. Bloch, Y. Yamamoto. Science, 298, 199 (2002).

[11] I. Shelykh, K.V. Kavokin, A.V. Kavokin, G. Malpuech, P. Bigenwald, H. Deng, G. Weihs, Y. Yamamoto. Phys. Rev. B, 70, 035320 (2004).

[12] B. Pietka, D. Zygmunt, M. Kro, A.A. Molas, A.A. Nicolet, F. Morier-Genoud, J. Szczytko, J. Lusakowski, P. Zieba, I. Tralle, P. Stepnicki, M. Matuszewski, M. Potemski, B. Deveaud. Phys. Rev. B, 91, 075309 (2015).

[13] C. Sturm, D. Solnyshkov, O. Krebs, A. Lemaitre, I. Sagnes, E. Galopin, A. Amo, G. Malpuech, J. Bloch. Phys. Rev. B, 91, 155130 (2015).

[14] C. Schneider, A. Rahimi-Iman, N.Y. Kim, J. Fischer, I.G. Savenko, M. Amthor, M. Lermer, A. Wolf, L. Worschech, V.D. Kulakovskii, I.A. Shelykh, M. Kamp, S. Reitzenstein, A. Forchel, Y. Yamamoto, S. Höefling. Nature, 497, 348 (2013).
[15] P. Stepnicki, B. Pietka, F. Morier-Genoud, B. Deveaud, M. Matuszewski. Phys. Rev. B, 91, 195302 (2015).

[16] N.D. Vy, H.T. Cao, D.B.T. Thoai, H. Haug. Phys. Rev. B, 80, 195306 (2009).

Редактор Г.А. Оганесян

\section{Coherent condensate of polaritons in planar microresonators in magnetic field}

\author{
A.V. Chernenko ${ }^{+}$, A. Rahimi-Iman*4, J. Fisher*, \\ M. Amthor*, C. Shneider*, S. Reitzenstein* , \\ A. Forchel*, S. Höefling* \\ + Institut of Solid State \\ Russian Academy of Sciences, \\ 142432 Chernogolovka, Russia \\ * Technische Physik, Physikalisches Institut and \\ Wilhelm Conrad Röntgen Research Center for Complex \\ Material Systems, Universität Würzburg, \\ D-97074 Würzburg, Germany \\ $\triangle$ Department of Physics and \\ Materials Sciences Center, \\ Philipps-Universität Marburg, \\ D-35032 Marburg,Germany \\ $\checkmark$ Institut fur Festkörperphysik, \\ Technische Universität Berlin, \\ D-10623 Berlin, Germany
}

Abstract The quasi-resonantly pumped planar GaAs-based microcavity is investigated in magnetic field up to $5 \mathrm{~T}$ by measuring photoluminescence and the second order auto-correlation function $g^{(2)}(0)$ of the polarization-filtered ground-state photoluminescence under pulsed laser excitation. The circular-polarization degree of the non-equilibrium condensate demonstrates a monotonic decrease with increasing magnetic field, while no such polarization is observed for the low density polariton emission. Hereby, originally different values of the coherence degree for the circularly left and right polarized condensate at low fields approach each other with increasing fields and become comparable at $5 \mathrm{~T}$. This effect directly corresponds to the change of the circular polarization degree of the condensate emission. 